\title{
Frequência de dores osteomioarticulares em profissionais do transporte público de São Luis-MA
}

\author{
Frequency of pain musculoskeletal in transportation professionals public São Luis-MA \\ Lucidalva Alves Abreu' ${ }^{1}$, Sarah Tarcisia Rebelo Ferreira de Carvalho', Karla Virginia Bezerra \\ de Castro Soares ${ }^{1,2}$, Ana Lourdes Avelar Nacimento ${ }^{1}$, Paulo Henrique Martins de Sousa ${ }^{1}$, \\ Maria Claudia Gonçalves ${ }^{1}$.
}

Resumo: Introdução: Os profissionais que trabalham com o transporte coletivo podem ser afetados por agentes físicos, químicos e biológicos que levam a implicações nos âmbitos orgânico-psicológico, podendo ocasionar múltiplas manifestações no sistema musculoesquelético, as patologias relacionadas à saúde ocupacional parecem estar intimamente ligadas a fatores intrínsecos ao ambiente do trânsito. Objetivo: Estimar a frequência de dores osteomioarticulares em motoristas do transporte público da cidade de São Luís - MA. Material e Método: Trata-se de um estudo de caráter quantitativo de delineamento transversal, a ser realizado com uma amostra de 38 cobradores e motoristas de uma empresa de ônibus atuante na cidade de São Luís-MA. Os dados foram obtidos através de questionários abordando informações sócio demográficas e ocupacionais dos participantes e também do Questionário Nórdico de Sintomas Osteomusculares (QNSO). As variáveis qualitativas foram apresentadas por meio de frequências absolutas e percentuais. Os dados foram digitados e analisados no SPSS 18.0. Resultados: A frequência de dores osteomioarticulares é de $92 \%$ em profissionais do transporte coletivo de São Luís-MA. O perfil é majoritariamente do gênero masculino (63\%), com idade média de 47,9 anos, sendo que $87 \%$ possui ensino médio completo e $61 \%$ estado civil casado. Os fatores mais estressantes no desempenho da atividade do transporte público é o transito (42\%). Dentre os profissionais apenas $29 \%$ relataram praticar algum tipo de atividade física, enquanto que dentre as doenças pregressas a mais prevalente vou a hipertensão arterial (42\%). A região lombar foi a mais frequente dentre os motoristas que relataram algum tipo de dor (85\%). Conclusão: Pode-se concluir que é elevado o número de profissionais que são acometidos pela sintomatologia dolorosa e que a região lombar é o principal sítio de acometimento.

Palavras-chave: Dor musculoesquelética, trabalho, saúde ocupacional.

Abstract: Introduction: There are a load of physical, chemical and biological agents involving in organizational and psychological spheres of professionals working with public transportation, causing multiple manifestations in body systems, and issues related to occupational health of these professionals live in a complex network of harmful factors which are intrinsic to the traffic environment. Objective: To estimate the frequency of musculoskeletal pain public transportation drivers in the city of Sao Luis - MA. Methodology: This is a quantitative character study, descriptive, observational cross-sectional study, to be conducted with a sample of 38 collectors and drivers of an active bus company in the city of Sao Luis, MA. Data were collected through questionnaires addressing socio demographic and occupational information of the participants and also the Nordic Musculoskeletal Questionnaire (QNSO). The qualitative variables were presented using absolute and percentage frequencies. Data were entered and analyzed using SPSS 18.0. Results: The frequency of osteoarticular pain is $92 \%$ in professional public transportation of São Luís-MA. The profile is predominantly male (63\%) with a mean age of 47,9 years, and $87 \%$ have completed high school and. $61 \%$ being married. The most stressful factors in the performance of public transport activity is the traffic (42\%). Among professionals, only $29 \%$ reported performing some type of physical activity, while among the previous diseases the most prevalent hypertension (42\%). The lumbar region was the most frequent among drivers who reported some type of pain (85\%). Conclusion: It can be concluded that there is a high number of professionals who are affected by the painful symptoms and the lower back is the main site of involvement.

Keywords: Musculoskeletal pain, labor, occupational health.

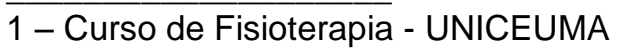




\section{Introdução}

A percepção de saúde e qualidade de vida, ao longo dos tempos, passa a ser analisada e compreendida conforme o processo histórico, sendo definida de acordo com o contexto sociocultural, político e econômico ${ }^{1}$. A noção de saúde que consta na preliminar da Constituição da Organização Mundial de Saúde (OMS), considera que: "saúde é o estado do mais completo bem estar físico, mental e social e não apenas a ausência de enfermidade", tornandose um direito fundamental do indivíduo social $^{2}$.

As condições de efetivação do processo saúde-doença estão diretamente relacionadas ao ambiente físico no qual o indivíduo está inserido, bem como a efetivação de políticas públicas de justiça social, alimentação, moradia e condições de trabalho ${ }^{3}$. Nos grandes centros urbanos, pode-se constatar um ambiente caótico nas vias públicas, congestionamentos, acidentes de trânsito, ruídos e estresse, bem como, a exposição a longas jornadas de trabalho e salários baixos $^{4}$. São fatores que precarizam a relação indivíduo-trabalho e infere especialmente no cotidiano de profissionais que trabalham com transporte coletivo urbano, motoristas e cobradores, que estão susceptíveis a tensões diárias e perdas gradativas de qualidade de vida ${ }^{5}$.

A problemática relacionada à
saúde ocupacional desses profissionais reside em uma rede complexa de fatores nocivos que são intrínsecos ao ambiente do trânsito ${ }^{6}$. As tensões decorrentes do embate com passageiros, as normas próprias de regulamentação do trânsito, as intercorrências e falhas mecânicas no veículo, exposição a vibração e ruídos, vias congestionadas, acidentes e alterações físicas relativas a movimentos repetitivos e posturas viciosas durante a jornada. Há uma carga de agentes físicos, químicos e biológicos que implicam nos âmbitos orgânico-psicológico desses indivíduos, ocasionando múltiplas manifestações nos sistemas orgânicos e consequentemente impactos na saúde pública ${ }^{7}$.

No Brasil, alguns estudos têm apontado a ocorrência de comorbidades nesses profissionais, a hipertensão arterial, obesidade e doenças cardiovasculares são apontadas como prevalentes neste meio devido ao sedentarismo e uma alimentação diária rica em calorias e com baixo valor nutritivo ${ }^{8}$. Outro estudo aponta que devido à poluição sonora urbana, ruídos do veículo e ausência da devida proteção acústica, $31 \%$ dos motoristas de ônibus de Curitiba apresentaram algum grau de perda auditiva9. A exposição ao estresse diário e a cobrança advinda tanto das empresas quanto dos usuários do transporte público, tornam esses profissionais mais susceptíveis a comorbidades psíquicas, como insônia, ansiedade e depressão ${ }^{10}$.

Há estudos que constatam uma relação íntima entre a jornada de trabalho e as queixas de dor no aparelho locomotor, sendo que quanto maior a manutenção de uma postura fixa por longos períodos de tempo, maior a possibilidade de aparecimento de dores, sobretudo quando esta postura é exercida durante trabalho noturno ${ }^{11}$.

Mediante este quadro, as comorbidades osteomioarticulares são as mais preocupantes, devido suas elevadas prevalências em estudos recentes. Uma pesquisa realizada em um município do estado de São Paulo evidenciou uma prevalência de 65,7\% de dores musculares auto-referidas em motoristas, sendo os sítios mais afetados os ombros, joelhos e coluna 
vertebral $^{12}$, corroborando com este resultado, outro estudo encontrou um valor de $53 \%$ de dores musculoesqueléticas em motoristas, sendo que $38 \%$ relataram dor localizada na coluna lombar ${ }^{13}$. Posturas inadequadas por um longo período, movimentos repetitivos, inclinação do banco do motorista, sobrecarga na musculatura paravertebral e a obesidade estão diretamente relacionadas a dores na região lombar ${ }^{14}$.

Uma das consequências mais insignes das dores decorrentes do trabalho são as posturas antálgicas adotadas e em suas consequências, os desvios posturais, como corroborado por um estudo que apontou outras repercussões dessas manifestações no âmbito social, sexual e profissional, com perdas significativas de percepção geral de bem-estar ${ }^{12}$.

Partindo deste princípio, é imprescindível o fomento de estudos que abordem a saúde ocupacional, investigando o bem estar bio-psicosocial e da categoria dos profissionais do transporte coletivo urbano e os agravantes que permeiam 0 aparecimento de doenças ocupacionais no ambiente laboral desses profissionais. $O$ objetivo geral desse estudo foi estimar a frequência de dores osteomioarticulares em motoristas do transporte público. Os objetivos específicos foram: Descrever - perfil dos profissionais e suas respectivas condições de trabalho; Verificar a prática de atividade física e doenças pregressas entre os profissionais e Identificar estruturas mais frequentemente acometidas por dores osteomioarticulares.

\section{Material e Método}

O estudo foi realizado em uma empresa de transporte coletivo da cidade de São Luís - MA, onde foram selecionados aleatoriamente uma amostra de 38 profissionais do transporte coletivo, sendo que 20 destes exerciam o cargo de motorista de ônibus coletivo e 18 cobradores. Adotou-se como critérios de inclusão: possuir idade inferior a 60 anos de idade, exercer a função no transporte público a tempo igual ou superior a 1 ano. Foram excluídos deste estudo, indivíduos que exerçam mais de uma função ocupacional, com alterações posturais significativas, fraturas ou tenham passado por procedimento cirúrgico no último ano.

Os participantes foram abordados nos pontos finais das linhas de ônibus, local onde permanecem no intervalo entre as viagens, período em que foi explanado o objetivo do estudo e seus respectivos benefícios para categoria. Os indivíduos que se enquadraram dentro dos critérios de inclusão e exclusão deste estudo foram convidados a assinar um Termo de Consentimento Livre e Esclarecido (TCLE).

Como instrumentos, utilizou-se questionário de identificação elaborado pelas autoras do presente estudo, composto por questões sobre dados sociodemoGráficos (idade, estado civil, escolaridade), dados do trabalho (carga horária diária, satisfação, instrumentalização) e dados clínicos (doenças pregressas, prática de atividade física e tabagismo/alcoolismo).

As manifestações álgicas osteomioarticulares e suas respectivas áreas de afecção foram mensuradas através do Nordic Musculoskeletal Questionnaire (NMQ), traduzido e validado por Pinheiro et al. (2002) ${ }^{11}$, é composto por questionamentos acerca de regiões anatômicas, com opções SIM ou NÃO para quatro situações ocorridas nos últimos 12 meses em decorrência da dor nessas respectivas 
regiões: 1) Formigamento ou dormência; 2) Empecilho para realização de atividades do trabalho; 3) Procura de serviços de saúde? 4) "Algum problema nesta região nos últimos 7 dias?".

Este estudo foi desenvolvido respeitando às Normas estabelecidas na Resolução 466/12 do Conselho Nacional de Saúde com relação à Realização de Pesquisa em Seres Humanos. Os indivíduos foram devidamente informados e esclarecidos quanto à importância e objetivo da pesquisa e havendo aceitação para sua participação, assinaram o Termo de Consentimento Livre e Esclarecido. Foram garantidos a possibilidade de não participação na pesquisa ou desistência, a privacidade, confiabilidade e o anonimato dos participantes.

Inicialmente foi realizada uma análise descritiva. As variáveis quantitativas foram descritas por média e desvio padrão (média $\pm \mathrm{DP}$ ) ou mediana. As variáveis qualitativas foram apresentadas por meio de frequências absolutas e percentuais. Os dados foram digitados e analisados no SPSS 18.0. $\mathrm{Na}$ estatística descritiva as variáveis qualitativas são descritas através de frequências absolutas e relativas.

\section{Resultados e Discussão}

A amostra estudada foi constituída por 38 profissionais do transporte coletivo apresentavam-se em consonância com os critérios de inclusão e exclusão deste estudo, encontrou-se uma frequência de $92 \%$ $(n=35)$ de dores osteomioarticulares dentre estes profissionais nos últimos 12 meses (Gráfico 1).

A literatura subsidia este resultado, um levantamento epidemiológico realizado com motoristas de transporte público, encontrou uma prevalência de $81,8 \%$ (TAMRIN et al., 2014). Colaborando com esse resultado, um estudo com profissionais atuantes no transporte público em uma cidade do estado de São Paulo, utilizando o mesmo instrumento, encontrou uma prevalência menor, porém relevante, de $65,7 \%{ }^{12}$.

\section{Luís - MA}

Gráfico 1 - Frequência de dores em profissionais do transporte público de São

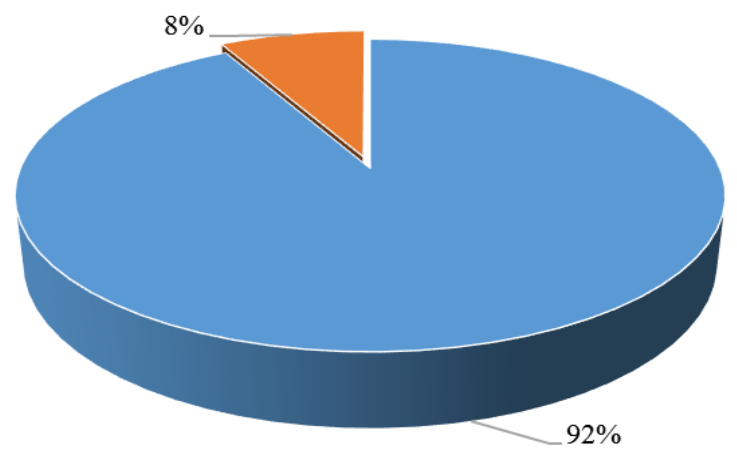

- Presença de Dor " Ausância de Dor 
Enquanto que para Carneiro et al. (2007) a frequência de dores constatada foi de $70,0 \%$ e $76,9 \%$ para motoristas e cobradores respectivamente. Outro estudo, obteve $61 \%$ de dores musculoesqueléticas em motoristas de ônibus urbano da cidade de Londrina - $\mathrm{PR}^{6}$.

A ausência de prevalências baixas na literatura demonstra que as condições ergonômicas

organizacionais que são submetidos esses profissionais incidem sobre a saúde e bem estar da maioria dos motoristas e cobradores de ônibus, as variações de valores encontrados ocorrem possivelmente devido aos instrumento utilizado para mensurar as dores, bem como das condições de trabalho ofertadas pelas empresas.

O perfil dos profissionais é composto majoritariamente por indivíduos do sexo masculino, a média de idade foi de 47,9 $( \pm 7,8)$, com a idade mínima de 29 e máxima de 60 anos. A maioria possui ensino médio completo e estado civil casado (Tabela 1).

A maioria dos profissionais de transporte de São Luís são do sexo masculino, corroborando com esta prédica $^{14}$, descreveu 0 perfil de profissionais do transporte público em que $98 \%$ pertenciam ao sexo masculino. Bem como em Pelotas RS, onde $78 \%$ dos trabalhadores de ônibus urbano são homens ${ }^{15}$. Para Pinto et al. $(2014)^{16}$, verifica que há uma divisão que vem sendo delineada, em que a função de motorista permanece quase que exclusivamente masculina e a de cobrador um espaço de apropriação feminina.

Tabela 1 - Perfil sócio-demográfico dos profissionais do transporte público de São Luís - MA.

\begin{tabular}{lcc}
\hline \multicolumn{1}{c}{ Variável } & $\mathbf{n = 3 8}$ & $\mathbf{( \% )}$ \\
\hline Gênero & & \\
Feminino & 14 & 36,8 \\
Masculino & 24 & 63,2 \\
Idade & & \\
$25-35$ & 2 & 5,3 \\
$36-45$ & 11 & 28,9 \\
$46-55$ & 17 & 44,7 \\
$>55$ & 8 & 21,1 \\
Escolaridade & & \\
Ensino Superior Incompleto & 5 & 13,2 \\
Ensino Médio Completo & 33 & 86,8 \\
Cargo exercido & & \\
Motorista & 20 & 52,6 \\
Cobrador & 18 & 47,4 \\
Estado Civil & & \\
Solteiros & 10 & 26,3 \\
Casados & 23 & 60,5 \\
Viúvos & 2 & 5,3 \\
Divorciados & 3 & 7,9 \\
\hline
\end{tabular}


A idade média foi de 49,7 anos, semelhante a média encontrada por outros estudos no Brasil 6,12,17. Em contraponto, Guterres et al. ${ }^{14}$ verificou uma média de 35,5 anos. Este dado sugere que há exigência de experiência e maturidade para a lida com os aspectos maçantes que 0 transito proporciona.

Quanto a escolaridade, a maioria da amostra relatou possuir ensino médio completo e uma minoria cursando 0 ensino superior, não havendo relatos de outras faixas de escolaridade. Este dado corrobora com as pesquisas encontradas na literatura ${ }^{14,15,18}$. Este dado é explicado devido ao próprio processo de admissão das empresas, onde possivelmente deve ser exigido 0 ensino médio como pré-requisito, a baixa procura pelo ensino superior ocorre possivelmente pelo conformismo diante da pouca possibilidade de promoção dentro da empresa.

Encontrou-se uma amostra predominantemente com estado civil casado, bem como evidenciado na $\begin{array}{ll}\text { literatura 6,12,14,19. Profissionais } & \end{array}$ casados tendem a inserir-se no meio produtivo e de profissões consideradas pesadas, em busca do sustento da família, sendo menos comum em indivíduos solteiros.

Quanto as condições de trabalho, a média da carga horária diária foi de 8,3 horas, variando de 6 a 10 horas, enquanto que a carga horária semanal média foi de 42,5 horas.

Ratificando este resultado, Costa et al. $^{18}$ verifica que na cidade de Joinville - SC a média de horas trabalhadas diariamente é de 8h20min, no entanto a carga horária semanal não ultrapassa as 40 horas. Enquanto que em Pelotas - RS a média é de 8,4 horas diárias e 44,5 semanais $^{15}$. Tal homogeneidade na carga diária ocorre devido a consolidação das leis que regulamentam as jornadas de trabalho, a divergência verificada na carga horária semanal ocorre devido ao fato de alguns profissionais pertencerem ao regime de 40horas e outros ao regime de 44 horas. 0 tempo médio de experiência no transporte público de São Luís foi de 10,5 anos $( \pm 3,6)$.

Alguns estudos encontraram variações não muito distantes deste valor, sendo que um estudo encontrou a média de 8,9 anos ${ }^{15,20}$, outro relata uma média de 9,6 anos ${ }^{6}$, enquanto que Pinto et al. $(2014)^{16}$ relata que 0 tempo médio de serviço no transporte público de Recife - PE é de 11,2 anos. Em contraponto a este dado, um estudo realizado na cidade de Curitiba - PR, identificou uma média de 6,3 anos ${ }^{9}$.

Possivelmente esta diferença é dada pelas diferenças estruturais das cidades, Curitiba possui um IDH maior que as cidades supracitadas e consequentemente maior oferta de emprego, o que possibilita maior rotatividade de funcionários na busca de melhores condições de emprego.

Este estudo encontrou um percentual de $66 \%$ de profissionais que relataram estar satisfeitos com 0 função exercida (Gráfico 2).

Colaborando com este dado, há uma pesquisa que relata $72 \%$ dos motoristas de ônibus sentem-se satisfeitos com o trabalho realizado ${ }^{17}$, no estudo de Pinto et al. $(2014)^{16}, 81 \%$ da amostra também relata satisfação com a tarefa desenvolvida. Contudo este dado não é consenso na literatura, visto que a maioria dos estudos apontam estatísticas negativas $^{6,8,15}$.

Uma possível hipótese é que a satisfação com 0 trabalho depende das condições e meios fornecidos pelo empregador para o bem estar do funcionário, bem como as condições 
Gráfico 2 -Satisfação profissionais do transporte público de São Luís - MA com a função exercida.

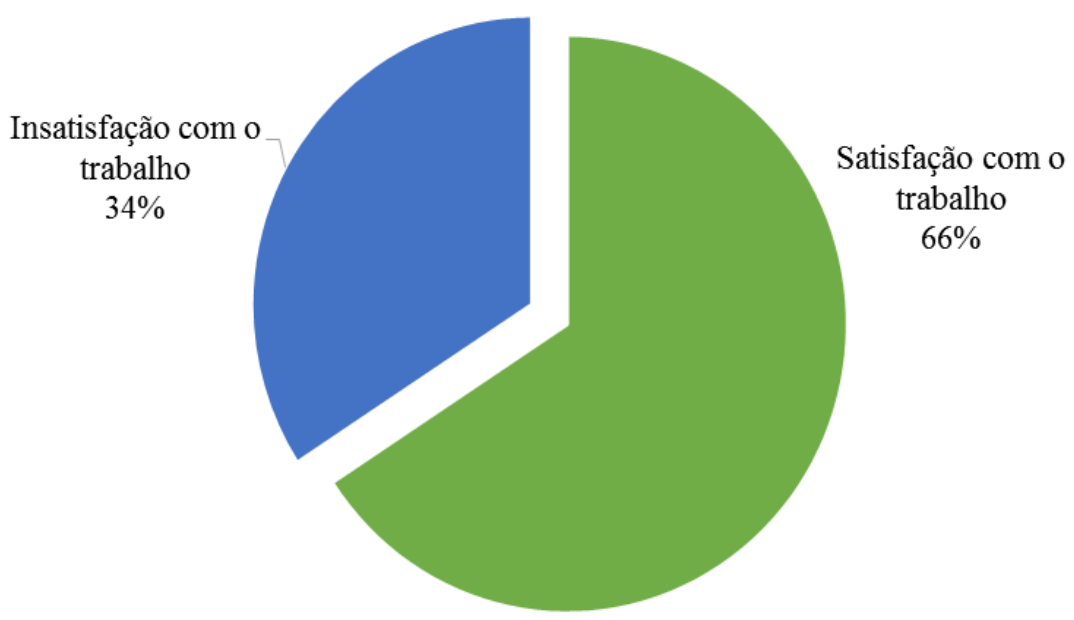

Gráfico 3 - Fatores estressantes em profissionais do transporte público de São Luís - MA.

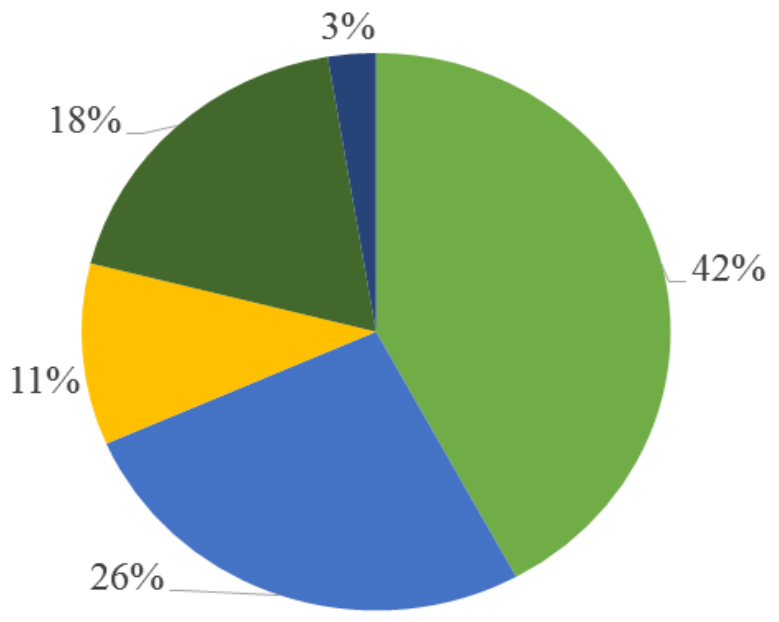

• Trânsito

- Insegurança

- Relação com

Passageiros

- Longa jornada/ Pausas curtas

- Baixa Remuneração

que este irá enfrentar na jornada de trabalho, portanto, há uma possibilidade de que as condições de trabalho fornecida por cada empresa e pela mobilidade urbana da cidade influencie nas diferenças de satisfação destes profissionais.

No que diz respeito ao fator mais estressante no exercício profissional, o trânsito foi a mais frequente resposta (Gráfico 3). 
Em outros estudos, o trânsito lidera as queixas de estresse e desgaste de motoristas ${ }^{15,16,21}$. A insegurança das grandes cidades e a interação com inúmeros indivíduos diariamente, torna estes profissionais suscetíveis ao adoecimento e a distúrbios psicológicos devido ao constante estado de alerta e fuga ${ }^{21}$.

Dentre os profissionais, apenas 11 (29\%) praticavam algum tipo de atividade física, sendo esta, a mais comum a caminhada $7(64 \%)$ e a prática de futebol 3 (21\%).

A literatura corrobora com este fato, sendo que em Joinville - SC, $28 \%$ dos profissionais praticavam algum tipo de atividade física ${ }^{18}$. Enquanto que outro estudo relata apenas $11 \%$ da amostra praticante ${ }^{6}$. Todavia, há estudos onde não se observa grandes diferenças ${ }^{12,14}$. Este resultado reflete a cultura sedentária que rege a sociedade contemporânea, bem como o tempo livre incipiente para a prática de atividades físicas, a diferença na literatura ocorre devido a sensibilização constante da população acerca dos benefícios da atividade físicas.

Quanto as doenças pregressas, a Hipertensão Arterial Sistêmica (HAS) foi a mais frequente, sendo relatada por $42 \%$ dos profissionais, conforme ilustrado pela tabela 2 .
Um dado que chama atenção neste estudo é que a história clínica da amostra evidenciou uma prevalência de $42 \%$ de hipertensos. Ratificando este resultado, Alquimim et al. $(2012)^{22}$, ao investigar o risco cardiovascular em profissionais de ônibus urbano, verificou que $51 \%$ dos investigados apresentaram valores acima dos limítrofes para pressão arterial sistólica. Em um estudo realizado em Teresina - PI, verificouse que $61,71 \%$ dos motoristas com a pressão arterial acima do considerado normal $^{20}$.

Este fenômeno ocorre possivelmente devido a composição da amostra, no que tange a idade, pertencendo a idade de risco para a HAS e no que tange ao gênero, visto que homens tem mais resistência para procurar serviços médicos.

No que se refere às estruturas acometidas pela sintomatologia dolorosa, a região lombar foi a mais frequente no número de queixas, tanto entre os motoristas $85 \%$, quanto entre os cobradores $83 \%$. Todavia a segunda estrutura mais acometida entre os motoristas foram os ombros $65 \%$, enquanto que entre os cobradores foi a região superior das costas 78\% (Gráfico 4).

Tabela 2 - Doenças pregressas em profissionais do transporte público de São Luís - MA.

\begin{tabular}{lcc}
\hline \multicolumn{1}{c}{ Doenças Pregressas } & $\mathbf{n}$ & $\%$ \\
\hline Hipertensão Arterial & 16 & 42,1 \\
Diabetes & 3 & 7,9 \\
Depressão & 3 & 7,9 \\
Obesidade & 3 & 7,9 \\
Tabagismo & 3 & 7,9 \\
Cardiopatias & 1 & 2,6 \\
Afecções do trato respiratório & 4 & 10,5 \\
Sem doenças pregressas & 5 & 13,2 \\
\hline
\end{tabular}


Gráfico 4 - Estruturas mais acometidas pela sintomatologia dolorosa profissionais do transporte público de São Luís - MA

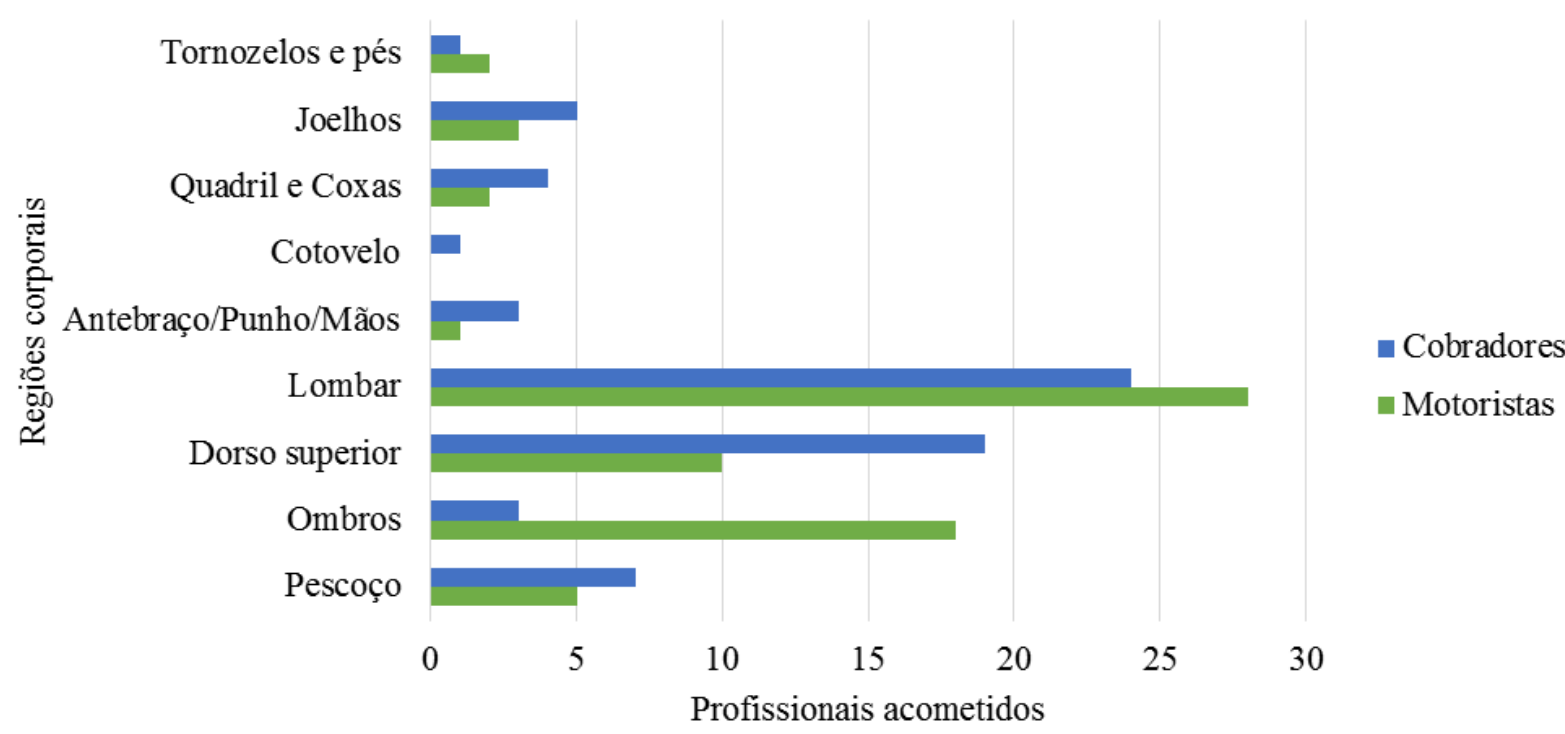

Este estudo identificou estruturas corporais mais frequentemente lesadas, sendo tanto em motoristas quanto em cobradores a região lombar foi a mais frequente, porém, em segundo lugar, o ombro foi mais frequente entre os motoristas, enquanto que a região dorsal superior entre os cobradores. A literatura atesta este dado, inclusive com estudos que analisam exclusivamente lombalgias nesses profissionais, para um estudo a lombalgia é maior em motoristas devido a angulação do assento e manutenção prolongada da postura sentada, enquanto que o cobrador exerce uma maior variabilidade de movimentos com maior liberdade de espaço ${ }^{23}$.

Segundo Guterres et al. $^{14}$, a região lombar é o sítio principal das dores em motoristas de ônibus, relatando uma prevalência de $57 \%$. Outro estudo, utilizando-se do mesmo instrumento que o presente, aponta a região lombar, seguida de ombros e joelhos como focos principais da sintomatologia dolorosa ${ }^{12}$. Este dado se confirma devido à ausência de suporte ergonômico nos ônibus, como assentos ajustáveis e apoio para as costas, quanto ao ombro, a exigência do ombro direito na utilização da marcha acarreta o desgaste de estruturas articulares e musculares, resultando na sintomatologia dolorosa.

\section{Conclusão}

Mediante a implementação do presente estudo, constatou-se que frequência de dores osteomioarticulares é alta em profissionais do transporte coletivo de São Luís.

O presente estudo possibilitou traçar o perfil destes profissionais, assim como as condições de trabalho a que são submetidos e a percepção destes indivíduos acerca dessas condições. 
Verificou-se que a maior parte da amostra não praticava atividade física e que a patologia pregressa que mais acomete esta população é a Hipertensão arterial.

A região lombar foi a mais frequente dentre os motoristas que relataram algum tipo de dor, bem como entre os cobradores, porém evidenciou-se altas prevalências de dores no ombro dentre motoristas e na região dorsal superior entre os cobradores.

Tendo em vista que no presente estudo, utilizou-se um número limitado de profissionais, sugerem-se trabalhos futuros, utilizando uma maior amostra e com análise estatística mais robusta.

\section{Referências}

1. MACEDO, A, C. et al. On the effects of a workplace fitness program upon pain perception: a case study encompassing office workers in a Portuguese context. Journal of occupational rehabilitation, v. $21, \mathrm{n}$. 2, p. 228-233, 2011.

2. BACKES, M. T. et al. Conceitos de saúde e doença ao longo da história sob 0 olhar epidemiológico e antropológico. Rev. enferm. UERJ, v. 17, n. 1, 2009.

3. ROCHA, S. V; ALMEIDA, M. M. G.; ARAÚJO T. M. Prevalência de transtornos mentais comuns entre residentes em áreas urbanas de Feira de Santana, Bahia. Rev Bras Epidemiol, v. 13, n. 4, p. 630-40, 2010.

4. ROSSI, A. M.; QUICK, J. C.; PERREWÉ, P. L. Stress e qualidade de vida no trabalho: 0 positivo e o negativo. In: Stress e qualidade de vida no trabalho: o positivo e o negativo. Atlas, 2009.

5. BARCZAK, R.; DUARTE, F. Impactos ambientais da mobilidade urbana: cinco categorias de medidas mitigadoras. Rev. Bras. Gest Urbana, Curitiba, v. 4, n. 1, p. 13-32, Jun., 2012.

6. CAVAGIONI, L. C. et al. Health problems, hypertension and predisposition to stress in truck drivers. Revista da Escola de Enfermagem da USP, v. 43, $\mathrm{n}$. SPE2, p. 1267-1271, 2009.

7. FASSA, A. G.; FACCHINI, L. A.; BREITENBACH, F. Prevalência de hipertensão arterial entre motoristas de ônibus em Santa Maria, Rio Grande do Sul. RBSO, v. 33, n. 118, p. 32-39, 2008.

8. GUARDIANO, J.; CHAGAS, T. Z.; SLOMP JUNIOR, H. Avaliação da perda auditiva em motoristas de ônibus de Curitiba. Revista CEFAC, v. 16, n. 1, p. 50-54, 2014.

9. ASSUNÇÃO, A. A.; SILVA, L. S. Condicoes de trabalho nos onibus e os transtornos mentais comuns em motoristas e cobradores: Regiao Metropolitana de Belo HorizonteMG. Cadernos de Saúde Pública, v. 30, n. 4, p. 899-899, 2013.

10. PINHEIRO, F. A. et al. Validação do Questionário Nórdico de Sintomas Osteomusculares como medida de morbidade. Rev Saúde Pública, v. 36, n. 3, p. 30712, 2002.

11. DE VITTA, A. et al. Sintomas musculoesqueléticos em motoristas de ônibus: prevalência e fatores associados. Fisioterapia em Movimento, Curitiba, v. 26, n. 4, p. 863-871, 2013.

12. LEMOS, L. C.; MARQUEZE, E. C.; MORENO, C. R. C. Prevalência de dores musculoesqueléticas em motoristas de caminhão e fatores associados. Revista Brasileira de Saúde Ocupacional, v. 39, n. 129, p. 26-34, 2014. 
13. GUTERRES, A. et al. Prevalência e fatores associados a dor nas costas dos motoristas e cobradores do transporte coletivo da cidade de Pelotas-RS. Revista Brasileira de Atividade Física \& Saúde, v. 16, n. 3, p. 240-245, 2012.

14. QUEIRÓGA, M. R.; MICHELS, G. B. A influência de características individuais na incidência de dor músculo-esquelética em motoristas de ônibus da cidade de LondrinaPR. Revista Brasileira de Atividade Física \& Saúde, v. 4, n. 2, p. 49-61, 2012.

15. SILVA, M. O. Pobreza, desigualdade e políticas públicas: caracterizando e problematizando a realidade brasileira. Revista Katálysis, v. 13, n. 2, p. 155-163, 2011.

16. CARNEIRO, L. R. V. et al. Sintomas de distúrbios ostemomusculares em motorista e cobradores de ônibus. Rev. bras. cineantropom. desempenho hum, v. 9, n. 3, 2007.

17. COSTA, M. M. et al. Excesso de peso em motoristas de ônibus da rede urbana. Revista Brasileira de Ciência e Movimento, v. 19, n. 1, p. 42-51, 2011.

18. TAMRIN, S. B. $M$. et al. Association of risk factors with musculoskeletal disorders among male commercial bus drivers in Malaysia. Human Factors and Ergonomics in Manufacturing \&
Service Industries, v. 24, n. 4, p. 369-385, 2014.

19. SILVA, A. M. B.; KELLER, B.; COELHO, R. W. Associação entre pressão arterial e estresse percebido em motoristas de ônibus. Rev Bras Epidemiol, v. 21, n. 4, p. 149-154, 2013. 21.

20. ASSUNÇÃO, A. A.; MEDEIROS, A. M. Violência a motoristas e cobradores de ônibus metropolitanos, Brasil. Rev Saúde Pública, v. 49, n. 1, p. 1-10, 2015.

21. ALQUIMIM, A. F. et al. Avaliação dos fatores de risco laborais e físicos para doenças cardiovasculares em motoristas de transporte urbano de ônibus em Montes Claros (MG). Ciência \& Saúde Coletiva, v. 17 , n. 8, p. 2151 2158, 2012.

22. MACEDO, C. S. G.; BATTISTELLA, L. R. Impacto da lombalgia na qualidade de vida: estudo comparativo entre motoristas e cobradores de transporte coletivo urbano. Revista Brasileira de Atividade Física \& Saúde, v. 6, n. 3, p. 82, 2012. 